\title{
Differing types of medical prevention appeal to different individuals
}

\author{
Nicolas Bouckaert $^{1} \cdot$ Erik Schokkaert $^{1,2}$
}

Received: 21 January 2015/ Accepted: 22 June 2015

(C) Springer-Verlag Berlin Heidelberg 2015

\begin{abstract}
We analyze participation in medical prevention with an expected utility model that is sufficiently rich to capture diverging features of different prevention procedures. The predictions of the model are not rejected with data from SHARE. A decrease in individual health decreases participation in breast cancer screening and dental prevention and increases participation in influenza vaccination, cholesterol screening, blood pressure screening, and blood sugar screening. Positive income effects are most pronounced for dental prevention. Increased mortality risk is an important predictor in the model for breast cancer screening, but not for the other procedures. Targeted screening and vaccination programs increase participation.
\end{abstract}

Keywords Screening - Vaccination - Prevention ·

Expected utility $\cdot$ Behavioral economics

JEL Classification D81 $\cdot$ I12

\section{Introduction}

Medical prevention, e.g., vaccination and screening, has become increasingly important in the health care systems of advanced countries. Health practitioners are concerned

Nicolas Bouckaert

nicolas.bouckaert@kuleuven.be

Erik Schokkaert

erik.schokkaert@kuleuven.be

1 Department of Economics, KU Leuven, Naamsestraat 69, 3000 Leuven, Belgium

2 CORE, Université catholique de Louvain, Louvain-la-Neuve, Belgium about the relatively low participation rates, even for prevention measures that are generally considered to be costeffective (such as influenza vaccination for the elderly and breast cancer screening for women between 50 and 69 years old). A careful look at this participation pattern reveals huge interindividual and intercountry differences. Moreover, participation also varies widely between different procedures for the same individuals.

Our main contribution to the literature is that we integrate existing evidence on participation in medical prevention. We test a rich model to see how different prevention procedures produce different results based on a variety of individual characteristics. First, the model compares both primary and secondary prevention. The former refers to interventions that aim at avoiding or reducing the occurrence of a disease (e.g., vaccination), and the latter to measures that aim at reducing the health consequences of a disease by detection and treatment in its early stages (e.g., cancer screening). Second, we distinguish between fatal (e.g., cancer) and non-fatal (e.g., dental caries) diseases. Third, our model takes individual health into account when analyzing why a patient chooses certain prevention procedures for certain diseases. In some cases, individuals may care more about the specific disease when their background health is worse (e.g., influenza), in other cases they may care more when their background health is better (e.g., dental caries). We show how these various possibilities change the comparative statics of the prevention decision and test the differential predictions with data from the Survey of Health, Ageing and Retirement in Europe (SHARE).

We stay in the tradition of the expected utility approach to study individual preventive medical behavior (see, amongst others: $[9,16,28,40])$, but we introduce a broad specification of utility (going beyond income and health) and interpret the parameters as individual-specific 
perceptions of reality. Indeed, taking a test imposes not only monetary (and time) costs, but also a psychological burden, which, according to the available surveys on motivations, may be crucial in explaining variations in preventive care participation (see, e.g., [37]). Moreover, while the literature has shown that subjective probabilities influence individual decisions, it has also become clear that the subjective risk perceptions covary only very partially with objective risk factors [8].

In our empirical work, we focus on six cases: breast cancer screening, dental caries screening, influenza vaccination, cholesterol screening, blood pressure screening, and blood sugar screening. These six procedures cover the range of interesting possibilities suggested by our model. We estimate probit models with the pooled data of the first two waves of SHARE. There have been previous empirical studies analyzing partly the same prevention procedures with SHARE data ([18, 22, 29] for influenza vaccination); ([18, 42, 43] for mammography); ([20, 21] for dental care). To the best of our knowledge, we present the first attempt to compare the results for the different procedures within a coherent theoretical approach, testing specific hypotheses about the differential comparative static effects. In accordance with the estimation strategies in Wübker [42] for breast cancer screening and Listl et al. [21] for dental care, we explain (part of) the intercountry differences through the introduction of institutional features that are specifically related to the prevention procedures analyzed. These specific features can be related to the parameters from our theoretical model. This approach appears more promising than controlling for general characteristics of a country's health care system [18]. ${ }^{1}$

The remainder of this paper is structured as follows. First, we introduce the model allowing for different types of disorders and characteristics of the process of medical prevention. Next, we derive comparative static results for the prevention decision. Third, we test the model hypotheses empirically. Most of our theoretical predictions are not rejected by the data and this result is robust to different specifications. The final section concludes and draws some policy implications.

\section{Model of medical prevention}

We propose an expected utility (EU) model that captures an individual's decision to participate in medical prevention for a specific disorder. Participation in prevention is taken to be a binary decision and is pursued when the

\footnotetext{
${ }^{1}$ None of the general characteristics used in Jusot et al. [18] turn out to have a significant effect for the explanation of influenza vaccination and breast cancer screening.
}

expected utility of participation exceeds the expected utility of non-participation, i.e., $\Delta \mathrm{EU}>0$, with

$$
\Delta \mathrm{EU}=\mathrm{EU}^{\text {participation }}-\mathrm{EU}^{\text {non-participation }}
$$

In what follows, we further detail this expected utility model as both participation and non-participation in prevention may lead to multiple potential health states. Rather than specifying a continuous value for the severity and survival rate of a disorder, we define several disease development stages and a binary distinction between fatal and non-fatal diseases. This simplification allows for a richer specification of the characteristics of the individual and the type of prevention. We will first work out the model for secondary prevention. Then, we will discuss primary prevention and show how it fits into the same model.

\section{Secondary prevention}

During each period ${ }^{2} t$, the individual derives utility $u($.) from income $y$, general background health represented by an index $h$ and prevention-specific health $m$. Utility is concave in income, with $u_{y}(y, h, m)>0$ and $u_{y y}(y, h, m) \leq 0$. A better general background health corresponds to a higher index score $h$ with $u_{h}(y, h, m)>0$ and $u_{h h}(y, h, m) \leq 0$. Variable $m$ represents the severity of the specific medical disorder for which the prevention procedure is set up. ${ }^{3}$ It takes one of four discrete values $(0<e<l<d)$, ranging from 0 , i.e., the individual does not suffer from the disorder, to $d$, the terminal stage of the disorder, in which the disorder cannot be treated anymore. The values $e$ and $l$ indicate early and late stages of the disorder, respectively. ${ }^{4}$ The stages are mutually exclusive.

\footnotetext{
2 In our model a "period" is defined as the normal amount of time in which an individual has to choose whether or not to participate in prevention. For influenza, a period is a 1-year interval, since an individual will have to decide to participate in prevention every year before the influenza season starts. For breast cancer screening on the other hand, the normal screening interval is 2 years. Furthermore, we assume for simplicity and clarity that this amount of time corresponds to the period in which a disease can develop into a severe illness that requires curative care, or in case of a fatal disease might result in death. While this is true for many diseases such as e.g., influenza, it is not always the case. The assumption can however be relaxed and our model adapted so that the prevention period and the period of disease development do not necessarily coincide. In this section, we drop the subscript $t$ for notational convenience.

${ }^{3}$ A natural interpretation of our model is that $h$ refers to the "health flow" corresponding to a Grossman-type health capital stock, and that $m$ refers to a specific health shock. Our model then assumes that the health (and hence utility) effects of a specific type of shock depend on its interaction with the general background health level.

4 The terminology "early" and "late" stage is just used as a convenient shortcut to indicate different levels of severity. Since we will later restrict ourselves to a two-period model, the timing of the treatment will not be analyzed in detail.
} 
The individual believes that she will develop the specific disorder with probability $p$. The out-of-pocket costs of treatment for the individual are $c_{e}$ and $c_{l}$ for, respectively, early and late stage treatment. They are independent of the individual's background health. If treated, the patient is cured of the illness, but relapse in a later period remains possible. The prevention behavior of the individual determines whether the disease develops into early or late stage.

Throughout the main analysis, we impose separability between utility from income and from health, i.e., $u(y, h, m)=v(y)+w(h, m)$. This assumption is widely used in the literature. ${ }^{5}$ In addition to utility from current income and health, the individual takes into account future utility $V_{t+1}$ which depends on the future streams of income and health. It is discounted with factor $\beta$ and corrected for the individual's mortality risk $\left(p_{x, t+1}\right)$ from any other cause but the prevention-specific disorder. The general utility specification that will reappear in each health state is then the following:

$v(y)+w(h, m)+\beta\left(1-p_{x, t+1}\right) V_{t+1}$

Our two-dimensional representation of health allows us to distinguish between different disorders in terms of their interaction with the general background health status. As will be explained later, we assume that the occurrence of a disorder (a shock) does not have a lasting effect on the background health $h$ in later periods if the disease is treated (and hence cured). At the moment of the shock, three types of disorders can be distinguished. The classification of different diseases in one of the three categories is ultimately an empirical matter, but we illustrate the three types with typical examples.

Complements Consider first the case of a minor medical problem, which does not strongly affect the background health of the individual: dental caries is an obvious example. In this case, it is natural to assume that "quality of the teeth" matters more for healthier individuals. This is represented in our model by

$w_{h}\left(h, m_{1}\right)<w_{h}\left(h, m_{2}\right), \quad \forall h \quad$ if $\quad m_{1}>m_{2}$

Comorbidities In the case of comorbidities, the occurrence of the disease has a stronger effect on health if background health is worse. A good example is influenza, since a healthy individual will suffer less from it than a sick individual, and runs a smaller risk of complications. If the

\footnotetext{
5 Income can be used for consumption goods that are complements to good health, e.g., travel, or substitutes for good health, e.g., assistance with self-care or a guide dog for the blind. The existing empirical results with respect to the sign and the magnitude of the cross-effect between health and income (or consumption) are inconclusive [14]. The results for the unrestricted utility function $u(y, h, m)$ are available from the authors on request.
}

utility loss due to the disorder is mitigated by a better initial health, this results in

$w_{h}\left(h, m_{1}\right)>w_{h}\left(h, m_{2}\right), \quad \forall h \quad$ if $\quad m_{1}>m_{2}$

Independence In principle, it is also possible that the effect of the new disorder is largely independent of the initial overall health status, resulting in

$w_{h}\left(h, m_{1}\right)=w_{h}\left(h, m_{2}\right), \quad \forall h, m_{1}, m_{2}$.

Perhaps an extreme diagnosis like that of a life-threatening cancer could be an example of independence, although in many cases comorbidities would be relevant for cancer also.

\section{Potential health states in case of non-participation}

The default situation is one where the individual does not participate in preventive care. Ex ante, she believes with a probability $1-p$ that she will be healthy and with a probability $p$ that she will be hit by the disorder. In the latter case, the disease will develop to the late stage, and there are two options. Either the disease is non-fatal and can be cured with treatment at a cost $c_{l}$, or the disease is fatal and cannot be cured (e.g., certain cancers), resulting in the individual's death. When the individual dies, we assume that she no longer benefits from current or future income. To that end, we introduce an indicator function $I(n f)$ that equals 1 if a disease is non-fatal and turns to 0 for fatal diseases. The expected utility in the non-participation case can therefore be written as

$\mathrm{EU}^{\text {non-participation }}=(1-p) u^{\mathrm{HE}}+p \times u^{S}$,

where the utilities in the healthy $(H E)$ and sick $(S)$ states are given respectively by

$$
\begin{aligned}
& u^{\mathrm{HE}}=v(y)+w(h, 0)+\beta\left(1-p_{x, t+1}\right) V_{t+1} \\
& u^{S}=I(n f)\left[v\left(y-c_{l}\right)+w(h, l)+\beta\left(1-p_{x, t+1}\right) V_{t+1}\right]
\end{aligned}
$$

\section{Potential health states in case of participation}

Secondary prevention allows early treatment of the disease $(m=e)$ at a lower cost of treatment $c_{e}<c_{l}$. Take breast cancer screening as an example. In the typical case, mammograms are used as screening technology. There are alternatives, such as self-examination of the breasts or examination of the breasts by the general practitioner (GP) or a more invasive breast tissue biopsy. Every screening technique entails different monetary, psychological (e.g., distress), physical (e.g., pain) and transaction costs (e.g., waiting and travel time). On the other hand, prevention can also induce positive emotions such as reassurance or relief. We indicate the intensity of the preventive procedure by 
$\alpha>0$, the out-of-pocket monetary cost by $c_{\alpha}$ and the psychic costs (net of psychic benefits) by $f(\alpha)$ (with $\left.\frac{\partial f(\alpha)}{\partial \alpha}>0\right)$.

If the test is positive, the early treatment is started. ${ }^{6} \mathrm{~A}$ true negative test rightly shows that an individual does not suffer from the disorder. In the case of a false-negative result, i.e., if the disorder is present but remains undetected, individuals end up in the late or terminal stage. The utility consequences of these different potential health states are as follows:

$u^{P}=v\left(y-c_{\alpha}-c_{e}\right)+w(h, e)+\beta\left(1-p_{x, t+1}\right) V_{t+1}-f(\alpha)$

$u^{T N}=v\left(y-c_{\alpha}\right)+w(h, 0)+\beta\left(1-p_{x, t+1}\right) V_{t+1}-f(\alpha)$

$u^{F N}=I(n f)\left[v\left(y-c_{\alpha}-c_{l}\right)+w(h, l)+\beta\left(1-p_{x, t+1}\right) V_{t+1}\right]-f(\alpha)$

where the superscripts $P, T N$, and $F N$ refer to "positive", "true negative" and "false negative", respectively.

The probabilities of ending up in the different health states depend on the effectiveness of the prevention program as measured by the test sensitivity $(s e \in[0,1])$. Test sensitivity is defined as the probability that a test will be positive for an ill individual and can be expressed in terms of the numbers of positive $\left(N^{P}\right)$ and false-negative tests $\left(N^{F N}\right)$ :

$s e=\frac{N^{P}}{N^{P}+N^{F N}}$

If the screening test gives perfect information we have $N^{F N}=0$ and $s e=1$.

We can then write the probabilities to end up in a certain health state in terms of $p$ and $s e$ :

$p^{P}=p \times s e$

$p^{T N}=(1-p)$

$p^{F N}=p(1-s e)$

If we combine the utility pay-offs and probabilities, we can formulate the expected utility in case of participation in a preventive care program:

\footnotetext{
${ }^{6}$ After a positive test result, a more conclusive second test (e.g., breast tissue biopsy) can reveal that the disorder was falsely suggested in the first round while the individual does not have the disorder. This is defined in the literature as a false-positive test. The frequency of false-positive results is captured by the test specificity, which is the probability that the test yields a negative result for an individual without the disorder. In order to simplify our analysis we abstract from the possibility of a second screening round. The results from a more complete model are similar. They can be obtained from the authors on request.
}

$$
\begin{aligned}
\mathrm{EU}^{\text {participation }} & =p \times s e \times u^{P}+(1-p) u^{T N} \\
& +p(1-s e) u^{F N}
\end{aligned}
$$

\section{The full model}

The individual will participate in prevention if $\Delta \mathrm{EU}>0$, with

$$
\begin{aligned}
\Delta \mathrm{EU}= & \mathrm{EU}^{\text {participation }}-\mathrm{EU}^{\text {non-participation }} \\
= & p \times s e \times u^{P}+p(1-s e) u^{F N} \\
& +(1-p) u^{T N}-p \times u^{S}-(1-p) u^{\mathrm{HE}} \\
=p \times & s e\left[u^{P}-u^{F N}\right]+p\left[u^{F N}-u^{S}\right] \\
& \quad+(1-p)\left[u^{T N}-u^{\mathrm{HE}}\right]
\end{aligned}
$$

It is useful to consider the preference ranking of the different states. For most realistic values of $f(\alpha)$, i.e., if psychological costs and distress due to prevention exceed psychological benefits from reassurance, and preventive care costs are not excessive, it is clear from Eqs. (7)-(11) that $u^{\mathrm{HE}}>u^{T N}>u^{P}$, and that $u^{S}>u^{F N}$. It is sufficient to assume that $u^{P}>u^{S}$ to get a full ranking. For fatal diseases (with $u^{S}=0$ ), this assumption boils down to the innocuous premise that taking an effective preventive action to avoid death yields a positive utility outcome $\left(u^{P}>0\right)$. For nonfatal diseases, we derive from Eqs. (8) and (9):

$$
\begin{aligned}
u^{P}-u^{S}= & \left(y-c_{\alpha}-c_{e}\right)-v\left(y-c_{l}\right) \\
& +w(h, e)-w(h, l)-f(\alpha)
\end{aligned}
$$

A positive value implies that the utility gain due to early discovery and treatment instead of late treatment is larger than the psychological costs related to prevention. In that case, we can conclude that:

$u^{\mathrm{HE}}>u^{T N}>u^{P}>u^{S}>u^{F N}$

If we accept Eq. (20), the first term in Eq. (18) is positive and represents the utility gain from a correct diagnosis and early preventive effort. Increasing sensitivity se leads to more effective prevention, and hence to a utility increase. The second and third term, on the other hand, are obviously negative. The second term indicates the utility loss due to a wrong screening diagnosis. The third term represents the utility loss for a healthy person when participating in prevention.

There are, however, two extreme cases: always-compliers and never-compliers. Some individuals enjoy prevention and will always comply. This is the case if psychological benefits exceed psychological costs and monetary prevention costs are limited. In this case $u^{P}>u^{F N}>u^{S}$ and $u^{T N}>u^{\mathrm{HE}}$, so that all three terms in Eq. (18) turn positive. On the other hand, it is possible that 
for some individuals, the psychological costs are prohibitively high, so that $u^{S}>u^{P}>u^{F N}$. This implies that $p \times u^{S}>p \times s e \times u^{P}+p(1-s e) u^{F N}$ and that the expected utility from prevention given by Eq. (17) is always negative. These individuals never comply with prevention. We exclude the extreme cases from the further analysis.

\section{Primary prevention}

The aim of primary prevention (for example immunization or Aspirin use to prevent cardiovascular diseases) is not to detect and treat a disease in its early stages but to reduce the probability of developing a disorder. The expected utility for non-participation in prevention is identical to the specification in Eqs. (6-8). In case the individual participates in primary prevention, two potential health states exist: the individual can either become sick or remain healthy. The former occurs when the preventive technology is not effective $(N E)$. A sick patient will be referred to latestage treatment (or will die if the disease is fatal). The utility pay-off and the probability of ending up in this state are:

$$
\begin{aligned}
u^{\mathrm{NE}}= & I(n f) \times\left[v\left(y-c_{\alpha}-c_{l}\right)+w(h, l)\right. \\
& \left.+\beta\left(1-p_{x, t+1}\right) V_{t+1}\right]-f(\alpha) \\
p^{\mathrm{NE}}= & p(1-e f)
\end{aligned}
$$

where $e f$ is a parameter that denotes the effectiveness of the primary preventive technology.

Alternatively, the individual remains healthy when she is not affected by the disease or when the preventive technology effectively protected her from contracting the disease. The utility pay-off and the probability of ending up in this effective state $(E)$ are:

$$
\begin{aligned}
u^{E} & =v\left(y-c_{\alpha}\right)+w(h, 0)+\beta\left(1-p_{x, t+1}\right) V_{t+1} \\
& -f(\alpha) \\
p^{E} & =1-p(1-e f)=(1-p)+p \times e f
\end{aligned}
$$

These expressions are very much in line with the specifications we have used to model secondary prevention. Eqs. (21) and (23) are identical to Eqs. (11) and (10), respectively, i.e., $u^{\mathrm{NE}}=u^{F N}$ and $u^{E}=u^{T N}$. If we reinterpret the sensitivity parameter for secondary prevention as the effectiveness parameter for primary prevention, i.e., if we take $s e=e f^{7}$, it follows that $p^{\mathrm{NE}}=p^{F N}$ and $p^{E}=p^{T N}+p^{P}$. In fact, the main difference between both types of prevention is that the positive test state with early treatment for secondary prevention is replaced by the true

\footnotetext{
7 Of course, this just means that the two parameters play the same role in both models, not that their numerical values are identical when comparing different procedures.
}

negative state in case of primary prevention. The expected utility for participation can then be written as follows:

$$
\begin{gathered}
\text { EU }^{\text {participation }}=[(1-p)+p \times e f] u^{E} \\
+p(1-e f) u^{\mathrm{NE}} \\
=\left[p^{T N}+p^{P}\right] u^{T N}+p^{F N} u^{F N}
\end{gathered}
$$

From Eq. (20), we know that $u^{T N}>u^{P}$. The replacement of $u^{P}$ by $u^{T N}$ in Eq. (26), shows that, ex ante and ceteris paribus, in comparison with secondary prevention, primary prevention makes it possible to realize an additional utility gain of the order $p^{P}\left(u^{T N}-u^{P}\right)$.

\section{Comparative statics of the prevention decision}

Individuals that do not expect to die in the immediate future will be confronted with multiple decision moments to participate in preventive care for a given disease. The same decision problem will return in each period until the uncertain moment of death. This means that the individual decides whether or not to participate in prevention in the current period, taking into account future utility and future preventive effort. To model this full process within a multiperiod setting is mathematically burdensome. We therefore focus on a simplified two-period model that is sufficient to yield the main insights. ${ }^{8}$

We assume that the individual lives during two periods and dies at the end of the second period. ${ }^{9}$ In period 1 , she decides whether or not to participate in the preventive program, while in period 2 , she does not participate in prevention and simply gets utility from income and health. The expected utility in period 2 is unaffected by individual behavior and is characterized as follows:

$$
\begin{aligned}
V_{2}= & \left(1-p_{2}\right)\left[v\left(y_{2}\right)+w\left(h_{2}, 0\right)\right] \\
& +I(n f) p_{2}\left[v\left(y_{2}-c_{l}\right)+w\left(h_{2}, l\right)\right]
\end{aligned}
$$

Implementing the decision rule, Eq. (1), for the first period gives for secondary prevention:

$$
\begin{aligned}
\Delta \mathrm{EU}_{1}= & p_{1} \times s e\left[v\left(y_{1}-c_{\alpha}-c_{e}\right)+w\left(h_{1}, e\right)\right. \\
& \left.+\beta\left(1-p_{x, 2}\right) V_{2}\right] \\
& +\left(1-p_{1}\right)\left[v\left(y_{1}-c_{\alpha}\right)-v\left(y_{1}\right)\right]-f(\alpha) \\
& +I(n f)\left[p_{1}\left(v\left(y_{1}-c_{\alpha}-c_{l}\right)-v\left(y_{1}-c_{l}\right)\right)\right. \\
& -p_{1} \times \operatorname{se}\left(v\left(y_{1}-c_{\alpha}-c_{l}\right)+w\left(h_{1}, l\right)\right. \\
& \left.\left.+\beta\left(1-p_{x, 2}\right) V_{2}\right)\right]
\end{aligned}
$$

\footnotetext{
${ }^{8}$ Results for a many-periods model can be obtained from the authors on request. If there are more periods, the issue of adherence to a prevention strategy can, in principle, become relevant (see, e.g., [10, 19]).

${ }^{9}$ If $I(n f)=0$, the patient dies at the end of the first period.
} 
and for primary prevention (with $s e=e f$ ):

$$
\begin{aligned}
\Delta \mathrm{EU}_{1} & =p_{1} \times \operatorname{se}\left[v\left(y_{1}-c_{\alpha}\right)+w\left(h_{1}, 0\right)\right. \\
& \left.+\beta\left(1-p_{x, 2}\right) V_{2}\right] \\
& +\left(1-p_{1}\right)\left[v\left(y_{1}-c_{\alpha}\right)-v\left(y_{1}\right)\right]-f(\alpha) \\
& +I(n f)\left[p_{1}\left(v\left(y_{1}-c_{\alpha}-c_{l}\right)-v\left(y_{1}-c_{l}\right)\right)\right. \\
& -p_{1} \times \operatorname{se}\left(v\left(y_{1}-c_{\alpha}-c_{l}\right)+w\left(h_{1}, l\right)\right. \\
& \left.\left.+\beta\left(1-p_{x, 2}\right) V_{2}\right)\right]
\end{aligned}
$$

Except for the first line, expressions (28) and $\left(28^{\prime}\right)$ are identical. We will now derive the comparative statics for the effects of the future, of background health and of income. We discuss briefly the results for the other parameters.

The future It follows from Eqs. (28) and (28') that

$$
\begin{aligned}
& \frac{\partial \Delta \mathrm{EU}_{1}}{\partial \beta}=(1-I(n f)) p_{1} \times s e\left(1-p_{x, 2}\right) V_{2} \geqslant 0 \\
& \frac{\partial \Delta \mathrm{EU}_{1}}{\partial p_{x, 2}}=-(1-I(n f)) p_{1} \times s e \times \beta \times V_{2} \leqslant 0 \\
& \frac{\partial \Delta \mathrm{EU}_{1}}{\partial V_{2}}=(1-I(n f)) p_{1} \times s e \times \beta\left(1-p_{x, 2}\right) \geqslant 0
\end{aligned}
$$

The future only influences the prevention decision in the case of a fatal disease, i.e., if $I(n f)=0$. Indeed, with a nonfatal disease, all the relevant consequences occur in the first period. For fatal diseases, prevention provides an opportunity to avoid death and thus increases the probability to benefit from future utility. Participation in prevention rises as the present value of the utility gain related to prevention increases. This happens when the level of future utility $V_{2}$ or the weight $\beta$ given to the future increase or the probability of dying from other causes $p_{x, 2}$ decreases.

Remember that we assume that an individual has a general background health $h_{2}$ in period 2, irrespective of being healthy, treated early or late in period 1 . This assumption can be relaxed to have e.g., a lower $h_{2}$ when treated late, due to permanent health damage. This leads to different utility values of $V_{2}$ depending on the potential states in period 1 . The consequence is that the future might matter also for non-fatal diseases, and, that the marginal effects (w.r.t. $\left.\beta ; p_{x, 2} ; V_{2}\right)$ go in the same direction as described for a fatal disease. ${ }^{10}$

\footnotetext{
${ }^{10}$ Another assumption that influences the comparative statics with respect to the future is that the frequency of prevention and the period of disease development coincide. If this is not the case, and e.g., prevention is recommended to be taken yearly while the disorder needs more than a year to develop to the late stage of the disorder, the prevention decision is taken in period 1 and potential curative treatment occurs in period 2. The consequence of this discrepancy is again that the future matters for a non-fatal disease, and that the marginal effects (w.r.t. $\left.\beta ; p_{x, 2} ; V_{2}\right)$ go in the same direction as described for a fatal disease.
}

Income The partial effect for current income $y_{1}$ can be derived from (28) for secondary prevention:

$$
\begin{aligned}
\frac{\partial \Delta \mathrm{EU}_{1}}{\partial y_{1}}= & p_{1} \times s e \times v_{y}\left(y_{1}-c_{\alpha}-c_{e}\right) \\
& +\left(1-p_{1}\right)\left[v_{y}\left(y_{1}-c_{\alpha}\right)-v_{y}\left(y_{1}\right)\right] \\
& +I(n f)\left[p_{1}\left(v_{y}\left(y_{1}-c_{\alpha}-c_{l}\right)-v_{y}\left(y_{1}-c_{l}\right)\right)\right. \\
& \left.-p_{1} \times s e \times v_{y}\left(y_{1}-c_{\alpha}-c_{l}\right)\right]
\end{aligned}
$$

and from $\left(28^{\prime}\right)$ for primary prevention:

$$
\begin{aligned}
\frac{\partial \Delta \mathrm{EU}_{1}}{\partial y_{1}}= & p_{1} \times s e \times v_{y}\left(y_{1}-c_{\alpha}\right) \\
& +\left(1-p_{1}\right)\left[v_{y}\left(y_{1}-c_{\alpha}\right)-v_{y}\left(y_{1}\right)\right] \\
& +I(n f)\left[p_{1}\left(v_{y}\left(y_{1}-c_{\alpha}-c_{l}\right)-v_{y}\left(y_{1}-c_{l}\right)\right)\right. \\
& \left.-p_{1} \times s e \times v_{y}\left(y_{1}-c_{\alpha}-c_{l}\right)\right]
\end{aligned}
$$

The first two lines in Eqs. (32) and (32') are always positive. The third and fourth lines are zero for fatal diseases and can be positive or negative for non-fatal diseases. Therefore for fatal diseases the overall income effect is always positive. If the disease is non-fatal, the sign of the overall income effect depends on the relative size of the underlying parameters.

A first-order Taylor expansion around $y_{1}$ allows us to formulate approximate conditions for $\frac{\partial \Delta \mathrm{EU}_{1}}{\partial y_{1}}$ to be positive. The results are summarized in Table $1 .{ }^{11}$ For non-fatal diseases, income has a positive effect if the (private) monetary costs of participation in prevention outweigh the savings in terms of curative treatment costs. If monetary costs are larger than monetary benefits, this will have a negative effect on the incentives for prevention, and, with a concave utility function, the negative impact will be more pronounced for poorer persons. This explains the positive income effect on participation in prevention. If costs are less than the benefits, an analogous reasoning yields a negative income effect. The conditions in Table 1 are easily interpreted. In most realistic cases of secondary prevention we may expect a positive income effect. If, for example, curative treatment and early treatment are equally well covered by health insurance, any monetary cost of prevention, as minor as it might be, leads to a positive income effect. In the case of primary prevention, the conditions for a positive income effect are stricter.

We can also draw conclusions about the effect of $y_{2}$ on the expected utility gain of taking a preventive test in period 1. It has a positive effect on participation, but only for fatal diseases. The obvious intuition is that an income increase enhances future utility $V_{2}$ and makes actual preventive effort more beneficial.

\footnotetext{
11 The details of the calculations are given in "Appendix 1".
} 
Table 1 Taylor conditions for positive income effect of $y_{1}$ on participation in period 1

\begin{tabular}{llll}
\hline Disease type & Fatal disease & Non-fatal disease, primary prevention & Non-fatal disease, secondary prevention \\
\hline Taylor condition $y_{1}$ & Always positive effect & $c_{\alpha} \geq p_{1} \times s e \times c_{l}$ & $c_{\alpha} \geq p_{1} \times s e \times\left(c_{l}-c_{e}\right)$ \\
\hline
\end{tabular}

General background health The comparative static expressions for background health $h_{1}$ are given by

$\frac{\partial \Delta \mathrm{EU}_{1}}{\partial h_{1}}=p_{1} \times s e \times w_{h}(h, e)-I(n f) p_{1} \times s e \times w_{h}(h, l)$

for secondary prevention and by

$$
\begin{aligned}
\frac{\partial \Delta \mathrm{EU}_{1}}{\partial h_{1}}= & p_{1} \times s e \times w_{h}(h, 0)-I(n f) p_{1} \\
& \times s e \times w_{h}(h, l)
\end{aligned}
$$

for primary prevention. The sign of these expressions depends on the type of illness. An overview of the different possibilities is given in Table 2. Note that these results offer an alternative explanation for the finding of $\mathrm{Wu}$ [41] that health has a positive effect on participation in breast cancer screening and a negative effect on influenza vaccination. To explain this discrepancy, Wu pointed at psychological factors such as fear and anxiety, varying discount rates by health status or differences in GP advice according to health status. Our model provides an easy explanation within the context of a standard expected utility model, based on the type of prevention and the disease characteristics. ${ }^{12}$

The effect of future health on participation in prevention is similar to the effect of future income. A better future background health makes it worthwhile to pursue prevention in the current period in the case of a fatal disease.

Other parameters The comparative statics for the other parameters can be found in "Appendix 2". Participation in prevention is unambiguously increased by lowering complexity $(\alpha)$, by lowering monetary $\left(c_{\alpha}\right)$ and psychological $f(\alpha)$ costs of prevention, by lowering early treatment costs $\left(c_{e}\right)$, and by enhancing the effectiveness of the preventive technology (se, ef). However as an increase in complexity $(\alpha)$ at the same time raises monetary costs $\left(c_{\alpha}\right)$ and improves effectiveness (se, ef), the positive and negative effects on participation should be weighed against each other. An increase in curative (late) treatment costs has no effect on preventive behavior for fatal diseases and an ambiguous effect for non-fatal diseases. For non-fatal diseases, the effect will be positive if the preventive technology is effective and the monetary costs of prevention are low, since in this case prevention provides a good alternative to curative treatment. A similar conclusion can

\footnotetext{
$\overline{12}$ Similar arguments are given in Maurer [22] and Mullahy [23].
}

Table 2 The expected effect of health on preventive action according to disease and prevention type

\begin{tabular}{lll}
\hline Disease type & Fatal disease & Non-fatal disease \\
\hline Complements $h$ and $m$ & Positive & Positive \\
Comorbidities $h$ and $m$ & Positive & Negative \\
Independence $h$ and $m$ & Positive & No effect \\
\hline
\end{tabular}

be drawn for risk perceptions $p_{1}$. They have a positive effect on participation for fatal diseases, but an ambiguous effect for non-fatal diseases, which will be positive for effective preventive procedures with low monetary costs.

\section{Empirical analysis}

For our empirical illustration, we analyze six types of disorders and their corresponding preventive care options: breast cancer, dental caries, influenza, hypertension, hypercholesterolemia and diabetes. In the next subsection we briefly describe the disorders and summarize the corresponding behavioral hypotheses. We then present the available data used in the empirical analysis. Finally, we present the results.

\section{Setup of the empirical exercise}

\section{Six procedures}

Breast cancer: fatal disease, secondary prevention, comorbidities or independence in health Breast cancer is the most common cancer among European women. It accounts for almost one in three new cancer cases and one in six cancer deaths. One in nine women develops breast cancer at some point in her life, and this fraction has increased over the years. It is possible to detect breast cancer and the chances of survival increase the earlier the cancer is treated. For this reason, many countries have set up a preventive screening program. Given the nature of breast cancer, we assume that late treatment of cancer results in death during the period. ${ }^{13}$

\footnotetext{
13 The American Cancer Society [1] distinguishes between 4 cancer stages. If breast cancer is detected and treated early (stage 1 or 2), the 5 year survival rate is nearly $100 \%$, whereas survival rates drop to $20 \%$ if cancer is detected in stage 4 .
} 
Dental caries: non-fatal disease, secondary prevention, complements in health The setup of preventive dental care is as follows. An (asymptomatic) individual visits the dentist preventively (without feeling pain or having dentalrelated problems). The dentist screens for dental caries and dental plaque. If the dentist observes irregularities action is undertaken. In the case of no prevention or a false-negative result, there will be curative treatment of the advanced dental problem. No government organized large scale preventive care programs exist and dental policies vary widely across European countries [38].

Influenza: non-fatal disease, primary prevention, comorbidities in health Influenza vaccination is one of the best-known and most studied examples of primary prevention. Influenza is a very common infectious disease that causes general discomfort for most and death for some. In the latter case, death is often the result of a weakening of the immune system caused by influenza and an additional infection, e.g., pneumonia. The US Centre for Disease Control and prevention (CDC) estimates that each year on average 5-20\% of the population suffers from seasonal influenza. The death rate from influenza in the period 1976-2007 is estimated between 1.4 and 16.7 deaths per 100,000 persons [31]. When combining this information, it is clear that the case fatality risk, which is the risk of dying when infected (or $1-I(n f)$ in our model), is far less than $1 \%{ }^{14}$ Therefore, we consider seasonal influenza to be a non-fatal disease.

Since the disease is infectious, immunization brings about positive externalities. Most developed countries provide subsidized vaccination programs for certain vulnerable groups within the population, such as chronically ill individuals or the elderly. In addition to government programs, a number of companies also provide vaccination programs.

Hypertension: non-fatal disease, secondary prevention, comorbidities in health Hypertension refers to chronic high blood pressure. In the US, 41 and $55 \%$ of non-institutionalized individuals aged above 45 or 65 , respectively, indicate to suffer from hypertension in 2012 [3]. Hypertension by itself is not life threatening and can be controlled and treated through dietary and lifestyle changes and/or medication. In combination with smoking, drinking or other chronic conditions, such as hypercholesterolemia and diabetes, the risk of dying from cardiovascular diseases increases strongly. ${ }^{15}$ Therefore, we assume comorbidities

\footnotetext{
$\overline{14}$ It should be noted however that mortality varies substantially by influenza virus type and age group. Most of the influenza- and pneumonia-related deaths occur among adults aged 65 or more. Hadler et al. [15] suggest case fatality rates per age group for seasonal influenza of $0.001-0.004 \%$ in the age group of $0-17$ years olds, $0.003-0.011 \%$ for adults between 18 and 64 and $0.11-0.44 \%$ for those aged 65 or more.
}

in health. There are two types of screening. First, individuals with a previous diagnosis of high blood pressure or of a cardiovascular condition are screened to follow closely their health status and evaluate the effects of treatment against hypertension. Second, asymptomatic individuals, i.e., individuals without increased risk for hypertension or cardiovascular diseases, have the possibility to opportunistically measure their blood pressure, e.g., as part of a medical check-up by the GP. Our model is relevant for the latter type of screening.

Hypercholesterolemia: non-fatal disease, secondary prevention, comorbidities in health Hypercholesterolemia refers to chronic high levels of cholesterol in the blood. In the US, 45 and $54 \%$ of the individuals aged above 45 or 65 , respectively, suffered from high cholesterol levels or took cholesterol-lowering medication between 2009 and 2012 [24]. High cholesterol levels put an individual at risk for, amongst other, heart diseases. Cholesterol levels can be lowered mainly by medication. There are no direct signs or symptoms of high cholesterol, so that screening, by means of a simple blood test, is required to ascertain its presence. As for hypertension, we focus on opportunistic screening by asymptomatic individuals.

Diabetes: non-fatal disease, secondary prevention, comorbidities in health Diabetes is a chronic disease that occurs when the pancreas does not produce enough insulin, or when the body cannot effectively use the insulin it produces, leading to high blood glucose levels. High blood glucose levels for prolonged periods cause damage to blood vessels, nerves and other tissues. This can lead to serious health complications such as cardiovascular diseases, stroke, blindness and kidney failure, especially in combination with disturbances in lipid metabolism, hypertension and smoking. The effects of diabetes can be mitigated for a substantial period of time by adjusting the diet, physical exercise, a healthy lifestyle, insulin injections and other medication. In our analysis, we focus on opportunistic screening by asymptomatic individuals.

\section{Hypotheses and empirical specification}

Participation in prevention is a discrete decision. In our theoretical model we assumed that individual $i$ participates if $\Delta \mathrm{EU}_{1}^{i}>0$, with $\Delta \mathrm{EU}_{1}^{i}$ given in Eqs. (28) and $\left(28^{\prime}\right)$. Adding a stochastic component $\varepsilon_{i}$ capturing idiosyncratic factors, missing variables and measurement errors, we can write the probability of participation as

\footnotetext{
15 Cardiovascular diseases are the main cause of death in Western countries. In the US, the incidence of heart diseases in 2012 is 18 and $30 \%$ for non-institutionalized individuals aged above 45 and 65 , respectively [3]. The mortality rate over the entire population in 2010 is 0.5 and $1.2 \%$, for individuals aged above 45 and 65 , respectively [24].
} 
Table 3 Overview of the theoretical hypotheses

\begin{tabular}{|c|c|c|c|c|}
\hline \multirow{2}{*}{$\begin{array}{l}\text { Effect on participation in } \\
\text { prevention (period 1) }\end{array}$} & \multicolumn{4}{|l|}{ Disorder } \\
\hline & $\begin{array}{l}\text { F, SP, Indep. (e.g., } \\
\text { breast cancer) }\end{array}$ & $\begin{array}{l}\text { NF, SP, Compl. (e.g., } \\
\text { dental caries) }\end{array}$ & $\begin{array}{l}\text { NF, PP, Comor. } \\
\text { (e.g., influenza) }\end{array}$ & $\begin{array}{l}\text { NF, SP, Comor. (e.g., hypertension, } \\
\text { diabetes, hypercholesterolemia) }\end{array}$ \\
\hline Decrease $h_{1}$ & Negative & Negative & Positive & Positive \\
\hline Increase $y_{1}$ & Positive & $\begin{array}{l}\text { Ambiguous (likely } \\
\text { positive) }\end{array}$ & $\begin{array}{l}\text { Ambiguous (likely } \\
\text { positive) }\end{array}$ & Ambiguous (likely positive) \\
\hline Increase $p_{x, 2}$ & Negative & No effect & No effect & No effect \\
\hline Decrease $\alpha, c_{\alpha}$ & Positive & Positive & Positive & Positive \\
\hline Increase $p_{1}$ & Positive & $\begin{array}{l}\text { Ambiguous (likely } \\
\text { positive) }\end{array}$ & $\begin{array}{l}\text { Ambiguous (likely } \\
\text { positive) }\end{array}$ & Ambiguous (likely positive) \\
\hline
\end{tabular}

$F$ fatal, $N F$ non-fatal, $S P$ secondary prevention, $P P$ primary prevention, Compl. complements, Comor. comorbidities, Indep. independence

$$
\begin{aligned}
P(i \text { participates }) & =P\left(\Delta \mathrm{EU}_{1}^{i}+\varepsilon_{i}>0\right) \\
& =P\left(\Delta \mathrm{EU}_{1}^{i}>-\varepsilon_{i}\right) .
\end{aligned}
$$

If we assume the random term to be normally distributed, this results in a standard probit model. The comparative static hypotheses about $\Delta E U_{1}$, as derived in the previous section, can then be rephrased directly as hypotheses on the probability of participation. ${ }^{16}$

To keep track of our theoretical predictions, it may be convenient to look at Table 3 , summarizing the hypotheses for the empirical cases that will be analyzed.

\section{Data}

Individual microdata are taken from SHARE. For breast cancer screening and influenza vaccination, we combine the microdata from SHARE with information from macrosources about the specific features of the prevention programs in the different countries. Table 4 gives an overview of the relevant data and shows how they are related to the variables in our theoretical model. Descriptive statistics are provided in Table 7 in "Appendix 3".

Individual data Our individual data come from the first (2004-2005) and second (2006-2007) waves of SHARE (version 2.5.0). SHARE is a micro-dataset, targeted at individuals aged 50 years and over (plus spouses). It covers more than 30,000 non-institutionalized individuals from 14 European countries and Israel. A household is selected in a random procedure, but with the specific requirement that at least one individual is aged 50 years or over. A full description can be found in [4].

\footnotetext{
$\overline{16}$ Belkar et al. [2] show that neglecting to distinguish between "aware" and "unaware" individuals may lead to a selection effect. However, they also show that the problem is not very serious if "censoring is modest and positive dependence between awareness and choice is substantial" (p. 44). This is likely to be the case with our data.
}

The dependent variables are binary variables that equal 1 if the individual has had a specific type of prevention in the last (two) year(s). The type of procedures include mammograms for women, preventive dental care, ${ }^{17}$ influenza vaccination, blood cholesterol test, blood pressure test and blood sugar test. Reported participation rates for our subsamples are 55, 42, 33, 52, 68, and $54 \%$, respectively. Data on participation in breast cancer screening and influenza vaccination were collected through a self-administered drop-off questionnaire. For breast cancer screening, we restrict our sample to women without a history of breast cancer. Since no respondent received the drop-off questionnaire in both waves, we are limited to a pooled cross-sectional analysis for breast cancer screening and influenza vaccination. In the case of dental prevention, an important number of individuals answered the question on participation in both waves. We account for this by pooling all observations while clustering error terms at the individual level. With respect to blood pressure, cholesterol and sugar tests, individuals are asked whether or not in the past year they were tested by a doctor or a nurse. This question was only surveyed in wave 2 through a self-administered drop-off questionnaire. ${ }^{18} \mathrm{We}$ focus on opportunistic screening by asymptomatic individuals, and exclude individuals with heart conditions and diabetes for

\footnotetext{
${ }^{17}$ We set preventive dental care equal to one if individuals reported visiting a dentist in the last 12 months for preventive use or prevention and treatment combined. The value is set to zero if the individual has not seen a dentist or has seen him/her only for treatment. Our empirical results are similar when using an alternative specification with a value equal to one if the dentist is contacted for prevention use only and zero otherwise.

18 All other SHARE data discussed below were collected using a computer assisted personal interviewing (CAPI) program. A selfadministered drop-off questionnaire can be biased, since lower socioeconomic groups tend to be underrepresented. Therefore, the answers to the drop-off questionnaire might not be representative of the population. However, Jusot et al. point out that prevalence rates obtained in the drop-off questionnaire correspond to available published OECD population data for most countries [18].
} 
Table 4 Overview of the data

\begin{tabular}{|c|c|c|c|c|}
\hline \multirow[t]{2}{*}{ Data } & \multicolumn{4}{|l|}{ Disorder } \\
\hline & Breast cancer & $\begin{array}{l}\text { Dental } \\
\text { caries }\end{array}$ & Influenza & $\begin{array}{l}\text { Other } \\
\text { disorders }\end{array}$ \\
\hline \multirow[t]{2}{*}{$h_{1}$} & \multicolumn{4}{|l|}{ Subjective health status } \\
\hline & \multicolumn{4}{|c|}{ Objective health variables: ADL, mobility, BMI, grip strength } \\
\hline$y_{1}$ & \multicolumn{4}{|l|}{ Equivalent household income, broadly defined } \\
\hline$p_{x}, 2$ & \multicolumn{4}{|l|}{ Mortality risk over period of 10 years } \\
\hline \multirow[t]{3}{*}{$\alpha, c_{\alpha}$} & \multicolumn{2}{|l|}{ Belonging to country target group for screening } & \multicolumn{2}{|l|}{$\begin{array}{l}\text { Belonging to country target group for } \\
\text { influenza }\end{array}$} \\
\hline & \multicolumn{2}{|l|}{ Probability of receiving an invitation letter } & \multicolumn{2}{|l|}{ Free or subsidized vaccination } \\
\hline & \multicolumn{4}{|l|}{ Population-based program completed } \\
\hline \multirow[t]{3}{*}{$p_{1}$} & Belonging to country target group for screening & Dentures & $\begin{array}{l}\text { Belonging to country target group for } \\
\text { influenza }\end{array}$ & \\
\hline & Past cancer diagnosis & $\begin{array}{c}\text { Trouble } \\
\text { biting }\end{array}$ & & \\
\hline & \multicolumn{4}{|c|}{$\begin{array}{l}\text { Age- and country-specific breast cancer incidence and } \\
\text { mortality rates }\end{array}$} \\
\hline $\begin{array}{l}\text { Control } \\
\text { var. }\end{array}$ & \multicolumn{4}{|c|}{ Education, nationality, gender, age, partner, smoker, house owner, country dummies by wave } \\
\hline
\end{tabular}

the subgroups of blood pressure and cholesterol screening. Furthermore, we exclude individuals with a history of hypertension or hypercholesterolemia, respectively, for the subgroup of blood pressure and cholesterol screening, as well as individuals who take specific medication for the disorder. For blood sugar screening, we exclude individuals with a history of diabetes or who use medication for diabetics.

As for the explanatory variables, we are particularly interested in variables that allow us to distinguish between the different disease and prevention combinations, i.e., health status and mortality risk. We supplement these with an income variable and various control variables.

SHARE contains subjective and objective health information. Self-assessed health is represented by four dummy variables, reflecting different perceived health levels: poor, fair, good, and, very good/excellent. In addition, we use two indices that capture a part of an individual's general background health in a more objective way: an index of limitations to six activities of daily living, ${ }^{19}$ and an index of limitations in mobility related to health. ${ }^{20}$ For each limitation a score of 1 is awarded. Subsequently the sum of all relevant limitations is rescaled (by the total number of

\footnotetext{
19 The activity questions that are used (yes/no): are you able to... dress?, walk across a room?, bathe or shower?, eat?, get in and out of bed? use the toilet?

20 The mobility questions that are used (yes/no): Are you able to... walk $100 \mathrm{~m}$ ?, get up from a chair after sitting for long periods?, climb stairs?, reach your arms above shoulder level?, carry weights over $5 \mathrm{~kg}$ ?
}

potential limitations) to a value between 0 and 1. Furthermore, we have information on the BMI and a score for the grip strength of the individual (ranging from 0 to 100). We use the average score out of two measurements with the individual's dominant hand.

The mortality risk $\left(p_{x, 2}\right)$ is captured by one question: "What are the chances that you will live to age $\mathrm{X}$ or more?". Age X is predetermined and depends on current age, e.g., for all individuals aged 65 or less, age $X$ is set at 75 , for individuals aged between 66 and 70, age $X$ is fixed at 80 , etc. To make mortality risk comparable across individuals, we estimate the probability of dying in the next 10 years using a Weibull specification to model longevity [17, 39]:

$$
F(x ; k ; \lambda)=1-e^{-\left(\frac{x}{\lambda}\right)^{k}}
$$

with $k$ the shape parameter or death rate, $x$ the time to death, and $\lambda$ the scale parameter. With the survival probability $(1-F(x ; k ; \lambda))$ and two age points, i.e., current age and age $\mathrm{X}$, we can compute $x$ and either $\lambda$ or $k$. In absence of additional information on survival probability (e.g., for another time span), we need a priori information on one of these two parameters. Longevity analysis using a Weibull specification suggests a death rate $k$ between 4 and 10 for individuals aged between 50 and 100 years [35, 39]. Therefore, in our empirical analysis we use $k=7$ as the baseline death rate and perform a sensitivity analysis for $k \in[4,10]$. The Weibull specification cannot meaningfully deal with certain survival probabilities of 0 or $100 \%$. However, since nobody can predict survival with certainty, 
we adjust 'certain' survival probabilities of 0 and $100 \%$ to slightly uncertain probabilities of 0.01 and $99.99 \%$. A similar approach has been followed in [28]. ${ }^{21}$

Income is interpreted as equivalent household income (using the square root equivalence scale), comprised of labor and retirement income as well as income from wealth (dividends, rental income etc.). We use reported (not imputed) income data and filter out households with zero or extremely high reported income. All amounts are expressed in euros using the exchange rates provided by SHARE, and subdivided into deciles across the different European countries (separately for each wave). ${ }^{22}$

There are a limited number of variables that relate to risk of a specific disorder. In the empirical analysis of breast cancer prevention, we take up an indicator for whether or not the individual has had a positive cancer diagnosis (other than breast cancer) in the past. We believe that this experience will increase the subjective belief (and/or objective risk) of developing breast cancer. The model for dental prevention is enlarged with a variable indicating whether or not the individual experiences biting problems and has dentures.

Control variables used in the empirical model are age (in classes of 5 years); education (based on ISCED-97 scale); ${ }^{23}$ dummies for gender, partner, house owner, nationality (native, EU-citizen or non-EU citizen), (past) smoker; and country dummies by wave. These control variables capture elements of awareness, prevalence, need, future utility, subjective beliefs and risk aversion.

Macro data Country dummies capture the effect of intercountry variation, but remain a black box with respect to the

\footnotetext{
21 There exists some doubt as to whether or not the answers to survival questions have predictive value for real longevity [33]. Moreover, skeptics point at a heaping of responses at focal-point values of 0,50 , or $100 \%$, hinting at biased response [6]. On the other hand, an individual has access to superior information about herself than is incorporated in a life table. For a discussion, see e.g., [26] or [43]. Peracchi and Perotti [26] using SHARE data and Smith et al. [30] using HRS data find evidence that subjective beliefs about longevity relate to observed survival patterns. For our purpose, it is not crucial whether or not individual beliefs are an accurate reproduction of reality, since the prevention decisions of individuals will be influenced by their subjective beliefs including biases.

${ }^{22}$ One of the reasons for using this decile information is that the income variable is defined differently in waves 1 and 2 of SHARE. In wave 1 it is reported before taxes and contributions, in wave 2 it is reported after taxes and contributions. To check the robustness of our findings we also introduced the two wave-specific income variables as separate variables in our regressions. In that way, we allow for the possibility that the income effects are different in the two waves (possibly because of the change in definition). Our empirical results and conclusions (see below) remain very similar (results available on request).

${ }^{23} \mathrm{We}$ create dummies for highest educational degree: primary education (ISCED 0-1), lower secondary education (ISCED 2), upper secondary education (ISCED 3-4), higher vocational education and university degree (ISCED 5-6).
}

underlying causes. While they are necessary when comparing countries (and are also present in our model), we enrich the SHARE data with information about health policies and health indicators from other sources. These can be seen as rough and partial measures of $\alpha$ and $c_{\alpha}$. The additional data are not individual specific but group or region specific. Due to missing data or lack of comparable information on health policies, Israel and Switzerland are left out of the analysis and only data from the 13 remaining countries are used (Austria, Belgium, Czech Republic, Denmark, France, Germany, Greece, Ireland, Italy, the Netherlands, Poland, Spain, Sweden). Since we keep the full set of country dummies in the model, the effects of all these additional policy and health variables are identified through the within-country variation.

For breast cancer, the WHO GLOBOCAN project provides age- and country-specific information on incidence and mortality rates for 2008 [36]. The rates are expressed in cases per 1000 individuals. The report on cancer screening in the European Union provides information on the type of screening program (population-based or opportunistic) ${ }^{24}$ and the implementation status ${ }^{25}$ [34]. In Germany, Denmark, and Italy, population-based programs are administered at a regional level, with varying progress in program implementation. We include the region-specific information on the implementation status of the breast cancer screening program in our dataset. Moreover, von Karsa et al. provide details on the country target group for screening and on the chances of receiving an invitation letter per country [34]. All of this information was matched with the characteristics of the individuals in our sample. ${ }^{26}$

\footnotetext{
${ }^{24}$ By population-based screening, we refer to an organized screening program (with a specified target group, a specific screening test, intervals, quality assurance, monitoring and other procedures) managed by an organization at a national or regional level. In addition to the high degree of organization, every eligible individual served by the screening program is individually identified and personally invited to attend screening. Opportunistic screening on the other hand refers to screening outside an organized program and without personal invitation. The initiative to perform a screening examination is taken either by the individual or the health care provider. Opportunistic screening may or may not be performed according to the public screening policy (if one exists), e.g., it may be applied to individuals outside the targeted population or according to a different screening technique.

25 It takes time to set up a population-based program. By implementation status, we refer to the progress made in this process. The starting point is a planning phase, followed by a pilot project, a rollout over the entire region/country and finally a completed populationbased screening program.

${ }^{26}$ We give some examples. If there are regional differences within a country (e.g., in implementation status of a screening program), this information is matched to the individuals living in these different regions. If a country implements a specific target group (e.g., women between 50 and 69 years old), a woman in that country belonging to that target group will get a value 1 for the corresponding variable, while the other women get a value zero.
} 
For Spain and Sweden, regional differences in target group definitions were taken into account.

Information on influenza vaccination policies and country differences can be found in the publications of the VENICE project [32]. ${ }^{27}$ Many countries define different target groups for influenza vaccination based on age (e.g., individuals aged 65 and over), on existing illnesses (e.g., individuals with chronic lung diseases) and on professions that have interactions with vulnerable groups (e.g., health care workers). We replicated the target groups based on the specific rules for each country. In addition, we distinguish between three reimbursement schemes: free vaccination, partially subsidized vaccination, or no subsidies.

Comparable regional or country information for the other disorders is limited or inaccurate, and is not included.

\section{Results}

Table 5 presents the averaged individual marginal effects of the participation determinants in the prevention of the different disorders. The analysis is performed on a large number of individuals: 11,350 individuals for breast cancer screening, 35,805 individuals for dental prevention of whom 12,972 have entries in both wave 1 and 2, 21,495 individuals for influenza vaccination, 4029 individuals for cholesterol screening, 3339 for blood pressure screening, and 5649 for

blood sugar screening. The analysis confirms results previously obtained with SHARE for breast cancer screening and influenza vaccination [18, 29, 42, 43], but some explanatory and control variables differ to match better our theoretical model. Of course, since all our data are basically taken from a cross section (and it is not possible to find convincing instruments), it would be wrong to give a causal interpretation to our results. Significant effects should be interpreted as associations. SHARE targets individuals aged 50 years and over (plus spouses), which implies that individuals below 50 years old are by definition partners and might not constitute a representative sample of the population. We keep all individuals in our sample regardless of age, but perform the empirical analysis also on the subsample of individuals aged 50 and over (see Table 8 in "Appendix 3"). The empirical results and conclusions are similar.

The results in Table 5 show that a decrease in background health status, represented by a higher BMI, ADL index or mobility index and a lower score on grip strength are either insignificant or have the hypothesized sign. The pattern that emerges from the self-assessed health dummies also confirms the hypothesis that a decrease in background

\footnotetext{
$\overline{27}$ VENICE is an acronym for Vaccine European New Integrated Collaboration Effort.
}

health decreases participation in breast cancer screening and dental prevention and increases participation in prevention for the other disorders. Even though our results are to be interpreted cautiously, it is very reassuring that the inclusion of multiple health variables, capturing divergent aspects of an individual background health, lead to partial effects that go in the same direction and that confirm the hypothesized correlations.

The marginal effects of self-assessed health are most pronounced for the types of prevention that can be provided by a general practitioner (GP), i.e., influenza vaccination, cholesterol screening, blood pressure screening, and blood sugar screening. An alternative explanation for these results could be that individuals with worse background health simply visit more often a GP, who might inform them about preventive actions. In this way, participation in prevention can be carried out in combination with a visit for another reason and individuals can be encouraged to take-up prevention in ways not directly captured in our model. We test whether this alternative channel is driving our results in two ways. First, we perform the baseline analysis on the subsample of individuals who have visited a GP in the past 12 months (results available on request). The effects for breast cancer screening, dental prevention and influenza vaccination are almost identical to the baseline results. With respect to the different blood tests, the significant effects among the objective indicators remain present and indicate that a decreasing health leads to a higher take-up of the test. The marginal effects of selfassessed health decrease in absolute value but the pattern remains visible and significant at a lower significance level.

Second, we introduce two additional explanatory variables to our baseline specification, i.e., the number of visits to the GP in the past 12 months and a GP quality index. We adopt the GP quality index as proposed by Wübker [43]. The GP quality index is not available for all individuals. Using it reduces the number of observations by more than $60 \%$ for dental prevention, by $22 \%$ for breast cancer screening and influenza vaccination and by around $8 \%$ for the other disorders. The results can be found in Table 9 in "Appendix 3". The inclusion of GP visits and GP quality reduces the point estimates of the marginal effects as well as their significance levels. Overall, the hypothesized correlations remain present, both for objective health indicators and for self-assessed health. Moreover, the results show that individuals who visited a GP more frequently in the past year are significantly more likely to participate in all types of prevention and that the quality of the GP has a positive effect on all types of prevention except dental prevention. These results stand to reason. Introducing the GP-variables does not alter considerably the overall pattern for the other variables (the most pronounced change is a decrease of the income effect for dental prevention). 


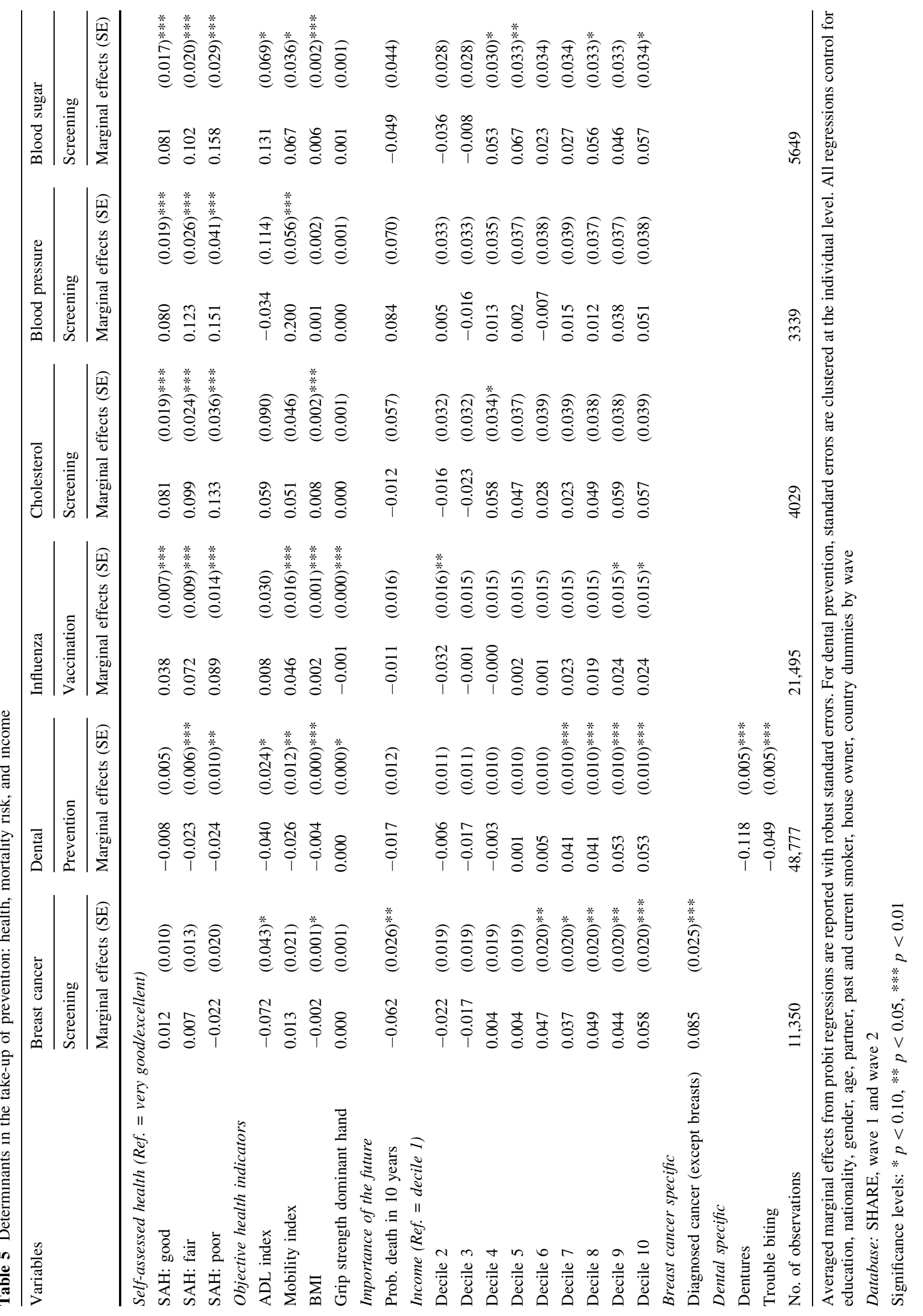


Also as expected, mortality risk over 10 years is an important predictor in the model of breast cancer screening, but has no significant effect on any other type of prevention. Sensitivity analysis confirms these results. The signs and significance do not change when we vary the death rate gradually from $k=4$ to $k=8.8$. For $k$ between 8.8 and 10 , the results are borderline significant with $p$ values between 10 and $15 \%$. Only in the model of blood sugar tests, an increase in mortality risk over 10 years with $4 \leq k<4.6$, slightly decreases the probability of participation at a significance level of $10 \%$. However, since SHARE is oriented towards individuals aged 50 and over, higher values for $k$, i.e., a more important increase in the probability of dying as one ages, are more probable.

Controlling for education levels, we find a positive effect of income on preventive behavior, although not always significant. We find that in particular deciles 1 to 3/4 participate less in prevention. The income effects are most pronounced for breast cancer screening and dental prevention. The former is again in line with the theoretical predictions. An alternative income specification, i.e., defining income deciles at the country level (by wave), does not change our results in any important way (results available on request).

Next, we have individual-specific information that serves as proxy for (subjective and/or objective) disease risk. We find that an earlier diagnosis of non-breast cancer increases the probability of participation in breast cancer screening on average by $8.5 \%$-points. Preventive dental care is negatively related to having dentures and having trouble biting.
This is not surprising, since the former probably reduces the need for regular preventive care, while the latter requires curative rather than preventive care.

Given that we test the effects of health, income and mortality risk across multiple prevention types, we may be confronted with the multiple testing problem, i.e., we run the risk of rejecting the null hypothesis too frequently. To correct for this, we applied the Holm-Bonferroni procedure. This method does not change any point estimate, but uses more stringent conditions to evaluate significance levels. We group (variable by variable) the preventive care types for which our hypotheses predict the same effect (see Table 3) and then apply the Holm-Bonferroni correction to this group. We give an example: for health variables a first group includes breast cancer screening and dental prevention for which we expect a negative effect, and the second group consists in the other prevention types, for which we expect a positive effect. The correction is applied within these groups. There are three main findings. First, income effects are no longer significant for influenza vaccination, cholesterol, blood pressure and blood sugar screening. This is, however, still consistent with our income hypothesis. Second, the effects of the ADL index on all types of prevention and the effect of the mobility index on blood sugar screening are no longer significant. Third, all other effects are unaffected. Overall, our main conclusions are confirmed even when adhering to more stringent significance criteria.

In Table 6, we add the additional information from macrosources to the microdata from SHARE. With the
Table 6 Determinants in the take-up of prevention: macro data on policy design

\begin{tabular}{|c|c|c|c|c|}
\hline \multirow[t]{2}{*}{ Variables } & \multicolumn{2}{|c|}{ Breast cancer screening } & \multicolumn{2}{|c|}{ Influenza vaccination } \\
\hline & \multicolumn{2}{|c|}{ Marginal effects (SE) } & \multicolumn{2}{|c|}{ Marginal effects (SE) } \\
\hline \multicolumn{5}{|l|}{ Breast cancer specific } \\
\hline Age- and country-specific incidence & -0.007 & $(0.018)$ & & \\
\hline Age- and country-specific mortality & 0.141 & $(0.058)^{* *}$ & & \\
\hline Country target group & 0.060 & $(0.024) * *$ & & \\
\hline Prob. receiving invitation letter & 0.172 & $(0.023)^{* * *}$ & & \\
\hline Pop.-based program complete & 0.179 & $(0.021)^{* * *}$ & & \\
\hline \multicolumn{5}{|l|}{ Influenza specific } \\
\hline Country target group based on age & & & 0.079 & $(0.017)^{* * *}$ \\
\hline Country target group based on illness & & & 0.058 & $(0.008)^{* * *}$ \\
\hline Subsidized vaccination & & & 0.085 & $(0.014)^{* * *}$ \\
\hline Free vaccination & & & 0.162 & $(0.014)^{* * *}$ \\
\hline
\end{tabular}

Averaged marginal effects from probit regressions are reported with robust standard errors. For dental prevention, standard errors are clustered at the individual level. All regressions control for health, mortality risk, income, education, nationality, gender, age, partner, past and current smoker, house owner, country dummies by wave. In addition, the regressions for breast cancer screening includes a dummy for having had a cancer (except breast cancer)

Database: SHARE, wave 1 and wave 2

Significance levels: $* p<0.10, * * p<0.05, * * * p<0.01$ 
exception of breast cancer incidence, all variables show very significant positive marginal effects. We observe that the participation decision is positively affected by age- and country-specific mortality rates. Breast cancer screening is higher for individuals who are targeted, who receive an invitation letter and who live in a country or region that has fully enacted a population-based program. The probability of taking up a vaccine increases if an individual belongs to a target group. Finally, we observe that monetary stimuli clearly increase the probability of receiving influenza vaccination, by $8.5 \%$-points for subsidized vaccination and by $16.2 \%$-points for free vaccinations. We conclude that policy features play a very important role in the takeup of prevention. They explain some of the intercountry variation without significantly altering the marginal effects of health, income, and mortality risk.

\section{Conclusions}

We analyzed participation in medical prevention with an expected utility model. Rather than focusing on one specific intervention, we aimed to explain the differences for various prevention procedures within one coherent model. This model is sufficiently flexible to distinguish primary and secondary prevention for either fatal or nonfatal diseases. Moreover, we integrated the idea of different disease types characterized by a different interaction with background health. The model yields different predictions in the different cases. We tested these predictions with individual data from SHARE and the model performed surprisingly well.

We believe that the construction of a flexible theoretical model is useful. It allows us to bring some coherency into the disparate insights from the empirical literature, and to validate differentiated hypotheses for different cases. ${ }^{28}$ In this respect, the expected utility model (broadly interpreted) seems to be an interesting starting point for further developments. These developments should go in three directions.

First, our theoretical model can be refined further. Introducing a richer dynamic structure would make it possible to integrate past behavior and more sophisticated expectations into the explanatory framework. ${ }^{29}$ More fundamentally, there are by now sufficient indications in the literature that the expected utility model cannot explain all the empirical regularities, not even when it is interpreted-

\footnotetext{
28 A similar position is taken by Howard [16, p. 893].

29 Some authors have used information in the third wave of SHARE to analyze the influence of reported past behavior in regard to (non)participation in breast cancer screening [42, 43] and in dental prevention [21].
}

as in our model-in a purely subjective way, taking due account of biases in the perception of costs and probabilities. Recent papers in the behavioral economic literature have built in other realistic features into the analysis of screening and prevention decisions: hyperbolic discounting and myopia [7, 12], loss-aversion over changes in beliefs [13], biased perceptions of risks in a rank-dependent utility model [11] and anticipatory feelings [25]. While some of these developments are very promising, it would be overly ambitious to try to build a general model of different prevention decisions incorporating these mechanisms. For a comparative exercise, the expected utility model remains a convenient and flexible starting point. However, it should be checked how much the more sophisticated behavioral models add to the explanatory power of an (extended) expected utility model, especially in cases of primary prevention and secondary prevention with screening as a necessary condition for treatment. ${ }^{30}$

Second, on the empirical side, we lack data on important parameters such as the subjective rate of time preference or the subjective perception of probabilities. Future work should try to collect direct measures of these parameters. ${ }^{31}$ Using such well-designed measures would allow a more convincing testing of the hypotheses.

Third, our model can also be used to draw policy conclusions. If the individuals perceive the correct values for all the variables in the model (costs, disease probabilities, quality of the testing procedure) the decision rule we derive gives, in principle, the criterion for welfare-optimizing behavior. However, as mentioned already in the introduction, there is growing evidence that individuals misperceive some of these variables. Maximizing "misperceived" expected utility will then lead to suboptimal behavior. Our results suggest which variables play the most important role in the real decision-making process of the patients and, hence, on which variables the regulator should focus if she wants to improve social welfare. This approach is analogous to the one that was followed by Phelps [27] on the basis of a very similar expected utility model. Rather than focusing on decisions by the patients (as we do), Phelps interprets his model as a description of provider behavior, but he also discusses the effects of potential errors in the basic underlying variables of the expected utility model.

Throughout the paper, we have focused on medical prevention procedures, mainly because participation is

\footnotetext{
30 The strongest arguments against using the expected utility model can be found in Oster et al. [25]. However, they analyze medical testing decisions for Huntington's disease-where at this moment no curative treatment is available.

${ }^{31}$ Examples in the literature are Bradford et al. [5] for time preferences and Carman and Kooreman [8] for subjective probabilities.
} 
modeled in a binary way, and we analyzed the prevention of a particular disorder, rather than an improvement of the general health status. Our model can be extended to include lifestyle choices, such as physical activity, eating healthy or (quitting) smoking, as long as the preventive decision can be defined in a binary way and the disorders that are prevented can be well delineated.

Acknowledgments Comments from Chiara Canta, Sverre Grepperud, Henri Ghesquiere, Tom Van Ourti, Erwin Ooghe, Geert Dhaene, Magne Mogstad, two anonymous referees, and participants at the Public Economics seminar in Leuven, the Ecore summer school and the EHEW 14 conference in Lyon are gratefully acknowledged. Nicolas Bouckaert is grateful to the Research Foundation-Flanders for providing a research fellowship. This paper uses data from SHARELIFE release 1, as of November 24th 2010 or SHARE release 2.3.1, as of July 29th 2010. The SHARE data collection has been primarily funded by the European Commission through the 5th framework programme (project QLK6-CT-2001- 00360 in the thematic programme Quality of Life), through the 6th framework programme (Projects SHARE-I3, RII-CT- 2006-062193, COMPARE, CIT5-CT-2005-028857, and SHARELIFE, CIT4-CT-2006-028812) and through the 7th framework programme (SHARE-PREP, 211909 and SHARE-LEAP, 227822). Additional funding from the U.S. National Institute on Aging (U01 AG09740-13S2, P01 AG005842, P01 AG08291, P30 AG12815, Y1-AG-4553-01 and OGHA 04-064, IAG BSR06-11, R21 AG025169) as well as from various national sources is gratefully acknowledged (see www.share-project.org/t3/ share/index.php for a full list of funding institutions).

\section{Appendix 1: First-order Taylor expansion}

We start from Eq. (32) and perform a Taylor expansion around $y_{1}$, for secondary prevention, this gives:

$$
\begin{aligned}
\frac{\partial \Delta \mathrm{EU}_{1}}{\partial y_{1}} & =v_{y}\left(y_{1}\right) p_{1} \times s e-v_{y y}\left(y_{1}\right)\left[c_{\alpha}\right. \\
& \left.\times\left(1-p_{1}(1-s e)\right)+c_{e} \times p_{1} \times s e\right] \\
& -I(n f)\left[v_{y}\left(y_{1}\right) p_{1} \times s e-v_{y y}\left(y_{1}\right)\right. \\
& \left.\times\left(c_{\alpha} \times p_{1}(1-s e)+c_{l} \times p_{1} \times s e\right)\right]
\end{aligned}
$$

and for primary prevention:

$$
\begin{aligned}
\frac{\partial \Delta \mathrm{EU}_{1}}{\partial y_{1}} & =v_{y}\left(y_{1}\right) p_{1} \times s e-v_{y y}\left(y_{1}\right)\left[c_{\alpha}\left(1-p_{1}(1-s e)\right)\right] \\
& -I(n f)\left[v_{y}\left(y_{1}\right) p_{1} \times s e-v_{y y}\left(y_{1}\right)\right. \\
& \left.\times\left(c_{\alpha} \times p_{1}(1-s e)+c_{l} \times p_{1} \times s e\right)\right]
\end{aligned}
$$

Since $v_{y}\left(y_{1}\right)>0$ and $v_{y y}\left(y_{1}\right) \leq 0$, the effect will always be positive for a fatal disease. For a non-fatal disease, we derive the following Taylor condition for secondary prevention:

$$
\begin{aligned}
& \frac{\partial \Delta \mathrm{EU}_{1}}{\partial y_{1}}=-v_{y y}\left(y_{1}\right)\left[c_{\alpha}+p_{1} \times s e \times\left(c_{e}-c_{l}\right)\right] \\
& \frac{\partial \Delta \mathrm{EU}_{1}}{\partial y_{1}} \geqslant 0 \Leftrightarrow c_{\alpha} \geqslant p_{1} \times s e \times\left(c_{l}-c_{e}\right)
\end{aligned}
$$

and for primary prevention:

$$
\begin{aligned}
& \frac{\partial \Delta \mathrm{EU}_{1}}{\partial y_{1}}=-v_{y y}\left(y_{1}\right)\left[c_{\alpha}-p_{1} \times s e \times c_{l}\right] \\
& \frac{\partial \Delta \mathrm{EU}_{1}}{\partial y_{1}} \geqslant 0 \Leftrightarrow c_{\alpha} \geqslant p_{1} \times s e \times c_{l}
\end{aligned}
$$

\section{Appendix 2: Comparative static results}

\section{Characteristics of the testing procedure}

Starting from Eq. (18), we derive for secondary prevention:

$$
\frac{\partial \Delta \mathrm{EU}_{1}}{\partial s e}=p_{1}\left(u^{P}-u^{F N}\right)>0
$$

where the conclusion about the sign follows from Eq. (20). An improvement of the effectiveness of prevention, without additional monetary or psychological costs, always makes prevention more attractive. For primary prevention, we have

$$
\frac{\partial \Delta \mathrm{EU}_{1}}{\partial s e}=p_{1}\left(u^{T N}-u^{F N}\right)>0 \text { for } e f=s e
$$

The comparative static results are straightforward for the "cost" parameters $\alpha$ and $c_{\alpha}$. We have $\frac{\partial u^{x x}}{\partial z}<0$, for $z=$ $\left(\alpha, c_{\alpha}\right)$ and for $x x=(P, T N, F N)$. We therefore conclude that

$$
\begin{aligned}
& \frac{\partial \Delta \mathrm{EU}_{1}}{\partial \alpha}<0 \\
& \frac{\partial \Delta \mathrm{EU}_{1}}{\partial c_{\alpha}}<0
\end{aligned}
$$

As could be expected, increased costs make preventive effort less attractive. If an increase in $\alpha$ leads to an increase in $c_{\alpha}$, the negative effects are reinforced. If, on the other hand, a policy change increases $\alpha$, and, at the same time, se, positive and negative effects should be weighed against each other.

\section{Characteristics of the disease}

The effect of a change in $p_{1}$ is less straightforward. Taking the derivative of Eq. (17), we get for secondary prevention:

$$
\begin{aligned}
\frac{\partial \Delta \mathrm{EU}_{1}}{\partial p_{1}} & =\left[u^{\mathrm{HE}}-u^{T N}\right]+\left[s e \times u^{P}\right. \\
& \left.+(1-s e) u^{F N}-u^{S}\right] \\
& =\left[v\left(y_{1}\right)-v\left(y_{1}-c_{\alpha}\right)\right] \\
& +\operatorname{se}\left[v\left(y_{1}-c_{\alpha}-c_{e}\right)+w\left(h_{1}, e\right)\right. \\
& \left.+\beta\left(1-p_{x, 2}\right) V_{2}\right] \\
& +I(n f)\left[v\left(y_{1}-c_{\alpha}-c_{l}\right)-v\left(y_{1}-c_{l}\right)\right. \\
& -s e\left(v\left(y_{1}-c_{\alpha}-c_{l}\right)+w\left(h_{1}, l\right)\right. \\
& \left.\left.+\beta\left(1-p_{x, 2}\right) V_{2}\right)\right]
\end{aligned}
$$


which has an obvious interpretation. The relative ranking of utility states in Eq. (20) shows clearly that if the individual is healthy (states $u^{\mathrm{HE}}, u^{T N}$ ), participation in prevention leads to additional costs and a utility loss, while if she is ill (states $u^{S}, u^{P}, u^{F N}$ ), it depends on the underlying parameters, such as the costs and the efficiency of the preventive procedures, whether prevention leads to a gain or a loss. As $p_{1}$ increases there is a shift away from the utility loss when healthy, towards the utility gain or loss when sick. The former leads to a positive effect on participation in prevention, captured by the first term in Eq. (35), while the latter may result in a positive or a negative effect on preventive behavior, captured by the second term in Eq. (35). The positive effect will dominate, i.e., $\frac{\partial \Delta \mathrm{EU}_{1}}{\partial p_{1}}>0$, for a fatal disease and for preventive procedures with a high sensitivity se and/or low screening $\operatorname{costs} c_{\alpha}$. For primary prevention (with $e f=s e$ ), the partial effect is similar, but $u^{P}$ is replaced by $u^{T N}$, which ceteris paribus leads to a higher marginal effect:

$$
\begin{aligned}
\frac{\partial \Delta \mathrm{EU}_{1}}{\partial p_{1}}= & {\left[u^{\mathrm{HE}}-u^{T N}\right]+\left[s e \times u^{T N}\right.} \\
& \left.+(1-s e) u^{F N}-u^{S}\right]
\end{aligned}
$$

We can also draw conclusions about the effect of $p_{2}$ on the probability of taking a preventive test in period 1 . As noted before, it will only have an impact for fatal diseases. In that case, we get from Eqs. (27) and (31) that

$$
\frac{\partial \Delta \mathrm{EU}_{1}}{\partial p_{2}}=-p_{1} \times s e \times \beta\left(1-p_{x, 2}\right)\left(v\left(y_{2}\right)+w\left(h_{2}, 0\right)\right)<0
$$

The intuition is obvious. Future utility $V_{2}$ unambiguously decreases as $p_{2}$ increases, since the individual is less likely to be healthy and more likely to be dead. As a result $\Delta \mathrm{EU}_{1}$ decreases and prevention becomes less interesting. This is in accordance with the conclusions from Eq. (31). ${ }^{32}$

\footnotetext{
32 If the time horizon is longer, as in the multi-period model, the effects of $p_{2}$ become more complex (results can be obtained from the author on request). Given that the individual can choose to participate in prevention in period 2 as well, she can counter partly the utility loss due to an increased risk of illness.
}

A last characteristic of the disease is the treatment cost, represented in the model by $c_{e}$ and $c_{l}$. Starting from Eqs. (28) and (28'), we get:

$$
\begin{aligned}
\frac{\partial \Delta \mathrm{EU}_{1}}{\partial c_{e}} & =-p_{1} \times s e \times v_{y}\left(y_{1}-c_{\alpha}-c_{e}\right) \leqslant 0 \\
\frac{\partial \Delta \mathrm{EU}_{1}}{\partial c_{l}} & =I(n f) \times p_{1}\left[v_{y}\left(y_{1}-c_{l}\right)\right. \\
& \left.-(1-s e) v_{y}\left(y_{1}-c_{\alpha}-c_{l}\right)\right]
\end{aligned}
$$

An increase in the cost of early treatment only matters for secondary prevention. It leads to a reduction in $\Delta \mathrm{EU}_{1}$ and, consequently, lowers the incentives for preventive action. Higher curative (late stage) treatment costs have no effect for fatal diseases, since no cure is available. For nonfatal diseases the effect is ambiguous, since the costs can occur both in case of participation (state $u^{F N}$ ) as in case of non-participation (state $u^{S}$ ). However, if se is high enough and/or $c_{\alpha}$ low, more expensive curative treatment increases the incentives for preventive effort. That was only to be expected. Prevention is the only possibility to avoid the larger cost, but this cost avoidance can only work if prevention is reasonably effective (se high enough) and screening costs are limited.

\section{Appendix 3: Additional empirical results}

See Tables 7, 8 and 9. 
Table 7 Descriptive statistics

\begin{tabular}{|c|c|c|c|c|}
\hline & Min & $\operatorname{Max}$ & Mean & Standard deviation \\
\hline Breast cancer screening & 0 & 1 & 0.540 & $(0.498)$ \\
\hline Dental prevention & 0 & 1 & 0.405 & $(0.491)$ \\
\hline Influenza vaccination & 0 & 1 & 0.327 & $(0.469)$ \\
\hline Cholesterol screening & 0 & 1 & 0.532 & $(0.499)$ \\
\hline Blood pressure screening & 0 & 1 & 0.694 & $(0.461)$ \\
\hline Blood sugar screening & 0 & 1 & 0.537 & $(0.499)$ \\
\hline Number of GP visits & 0 & 98 & 4.586 & $(7.173)$ \\
\hline GP quality index & 0 & 1 & 0.253 & $(0.234)$ \\
\hline SAH: very good or excellent & 0 & 1 & 0.290 & $(0.454)$ \\
\hline SAH: good & 0 & 1 & 0.381 & $(0.486)$ \\
\hline SAH: fair & 0 & 1 & 0.239 & $(0.427)$ \\
\hline SAH: poor & 0 & 1 & 0.090 & $(0.286)$ \\
\hline ADL index & 0 & 1 & 0.036 & $(0.134)$ \\
\hline Mobility index & 0 & 1 & 0.175 & $(0.268)$ \\
\hline BMI & 11 & 78 & 26.529 & $(4.436)$ \\
\hline Grip strength dominant hand (average of two attempts) & 0 & 91 & 29.551 & $(15.006)$ \\
\hline Prob. death in 10 years & $5.14 \mathrm{e}-08$ & 1 & 0.122 & $(0.211)$ \\
\hline Age below 40 & 0 & 1 & 0.002 & $(0.044)$ \\
\hline Age between 40 and 44 & 0 & 1 & 0.007 & $(0.082)$ \\
\hline Age between 45 and 49 & 0 & 1 & 0.026 & $(0.158)$ \\
\hline Age between 50 and 54 & 0 & 1 & 0.168 & $(0.374)$ \\
\hline Age between 55 and 59 & 0 & 1 & 0.185 & $(0.388)$ \\
\hline Age between 60 and 64 & 0 & 1 & 0.166 & $(0.372)$ \\
\hline Age between 65 and 69 & 0 & 1 & 0.145 & $(0.352)$ \\
\hline Age between 70 and 74 & 0 & 1 & 0.120 & $(0.325)$ \\
\hline Age between 75 and 79 & 0 & 1 & 0.091 & $(0.287)$ \\
\hline Age between 80 and 84 & 0 & 1 & 0.058 & $(0.234)$ \\
\hline Age 85 and over & 0 & 1 & 0.033 & $(0.180)$ \\
\hline Gender: male & 0 & 1 & 0.443 & $(0.497)$ \\
\hline Gender: female & 0 & 1 & 0.557 & $(0.497)$ \\
\hline Nationality: native & 0 & 1 & 0.980 & $(0.140)$ \\
\hline Nationality: EU citizen (not native) & 0 & 1 & 0.012 & $(0.111)$ \\
\hline Nationality: outside EU & 0 & 1 & 0.007 & $(0.086)$ \\
\hline Has partner & 0 & 1 & 0.748 & $(0.434)$ \\
\hline Schooling: primary & 0 & 1 & 0.325 & $(0.468)$ \\
\hline Schooling: lower secondary & 0 & 1 & 0.179 & $(0.384)$ \\
\hline Schooling: upper secondary & 0 & 1 & 0.305 & $(0.460)$ \\
\hline Schooling: university & 0 & 1 & 0.191 & $(0.393)$ \\
\hline House owner & 0 & 1 & 0.730 & $(0.444)$ \\
\hline Smoked in the past & 0 & 1 & 0.472 & $(0.499)$ \\
\hline Smokes currently & 0 & 1 & 0.197 & $(0.398)$ \\
\hline Diagnosed cancer (except breasts) & 0 & 1 & 0.039 & $(0.195)$ \\
\hline Breast cancer: incidence (per 1000 cases) & 0.077 & 5 & 2.794 & $(0.883)$ \\
\hline Breast cancer: mortality (per 1000 cases) & 0.012 & 2 & 0.777 & $(0.463)$ \\
\hline Breast cancer: target group & 0 & 1 & 0.724 & $(0.447)$ \\
\hline Breast cancer: prob. receiving invitation letter & 0 & 1 & 0.381 & $(0.444)$ \\
\hline Breast cancer: pop.-based program complete & 0 & 1 & 0.542 & $(0.498)$ \\
\hline Influenza: target group (age) & 0 & 1 & 0.528 & $(0.499)$ \\
\hline Influenza: target group (illness) & 0 & 1 & 0.259 & $(0.438)$ \\
\hline Influenza: subsidized vaccination & 0 & 1 & 0.334 & $(0.472)$ \\
\hline Influenza: free vaccination & 0 & 1 & 0.292 & $(0.455)$ \\
\hline
\end{tabular}

Database: SHARE, wave 1 and wave 2 


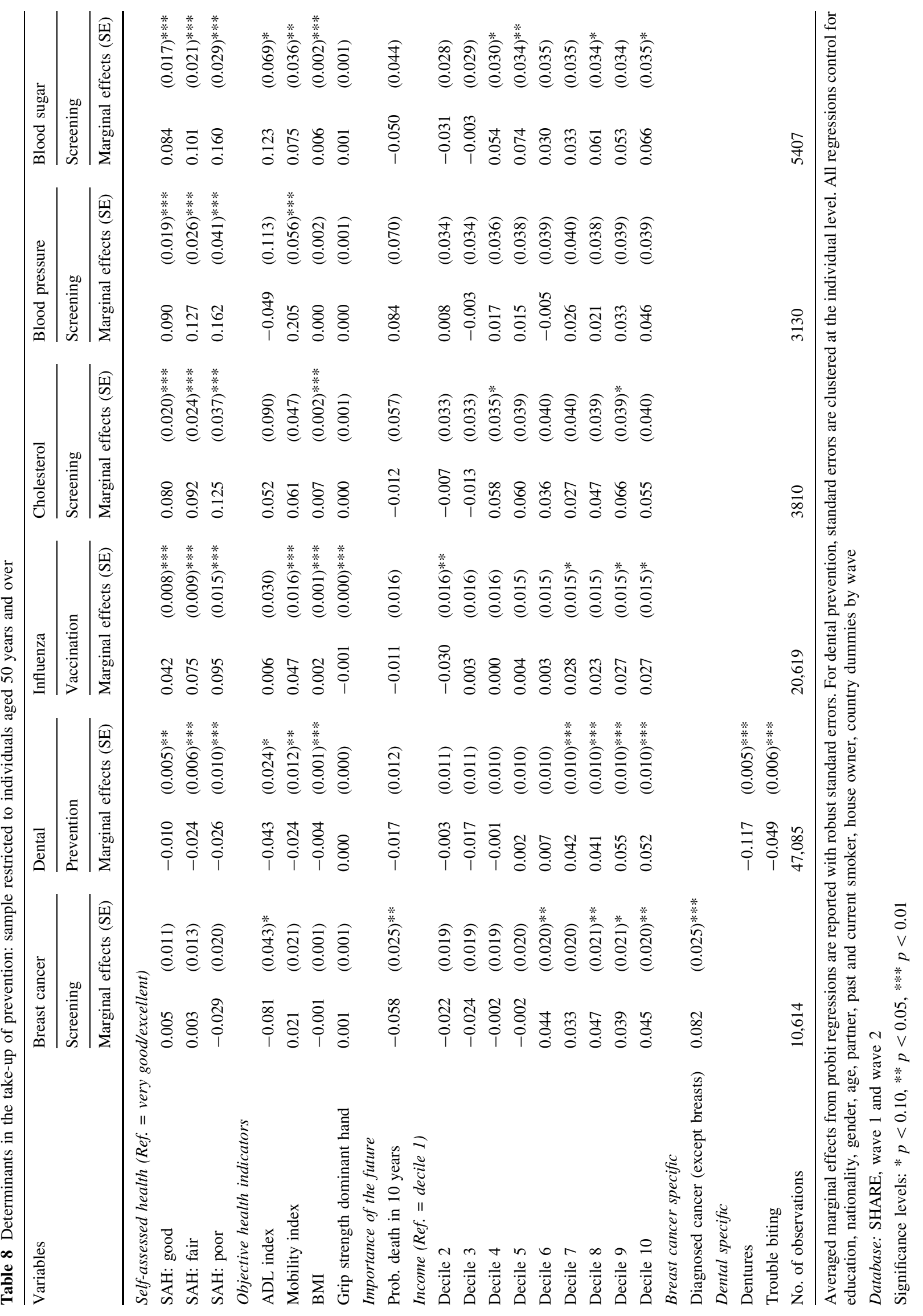




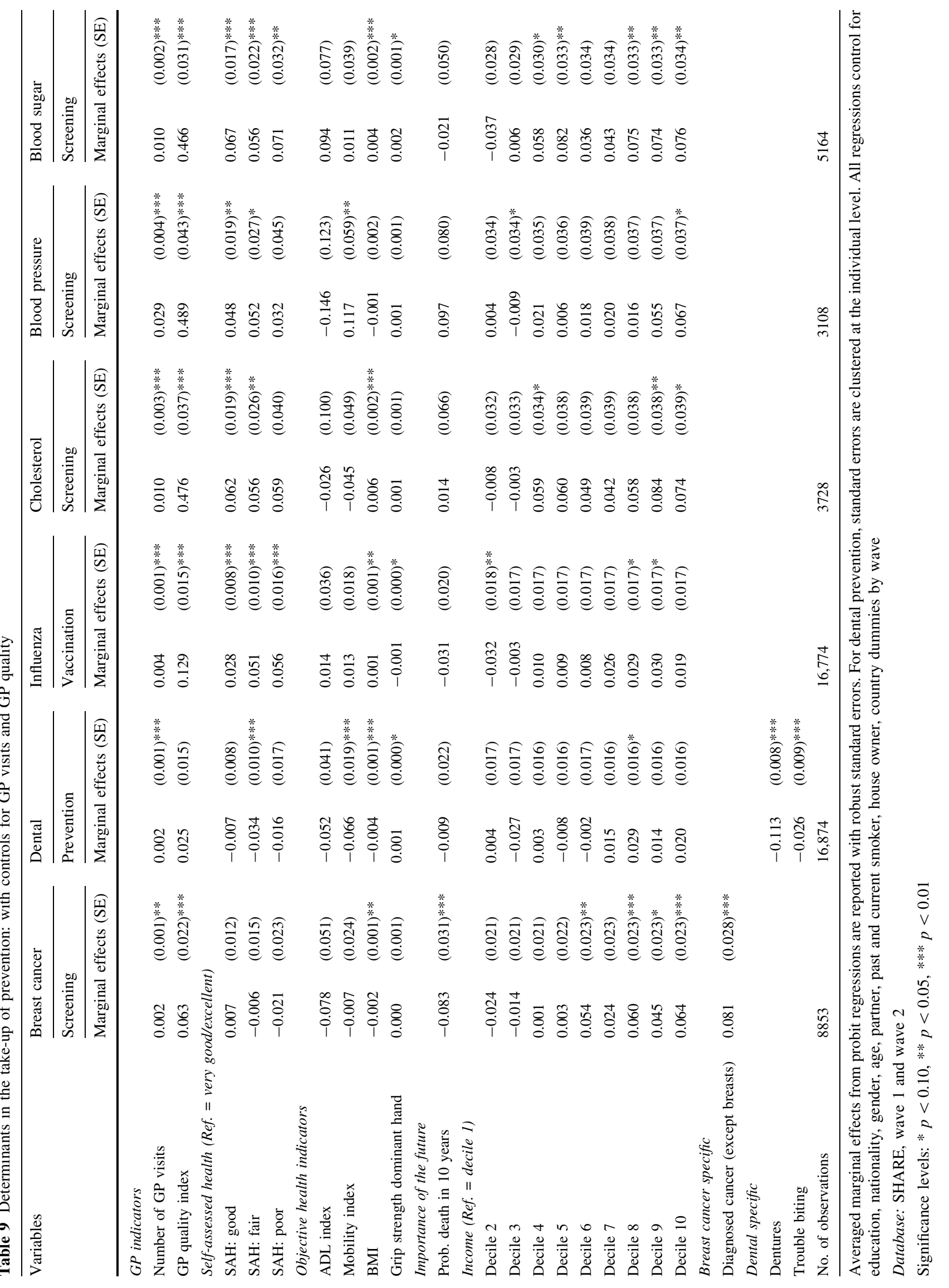




\section{References}

1. American Cancer Society: Breast cancer survival rates by stage. http://www.cancer.org/cancer/breastcancer/detailedguide/breastcancer-survival-by-stage (2013). Accessed 2 Dec 2013

2. Belkar, R., Fiebig, D., Haas, M., Viney, R.: Why worry about awareness in choice problems? Econometric analysis of screening for cervical cancer. Health Econ. 15, 33-47 (2006)

3. Blackwell, D.L., Lucas, J.W., Clarke, T.C.: Summary health statistics for U.S. adults: National Health Interview Survey, 2012. Vital Health Stat. 10(260), 1-161 (2014)

4. Börsch-Supan, A., Brugiavini, A., Jürges, H., Mackenbach, J., Siegrist, J., Weber, G.: Health, Ageing and Retirement in Europe. First Results from the Survey of Health, Ageing and Retirement in Europe, p. 370. Research Institute for the Economics of Aging, Mannheim (2005)

5. Bradford, W.D., Zoller, J., Silvestri, G.A.: Estimating the effect of individual time preferences on the use of disease screening. South. Econ. J. 76(4), 1005-1031 (2010)

6. Bruine de Bruin, W., Fischbeck, P.S., Stiber, N.A., Fischhoff, B.: What number is "Fifty-Fifty"?: Redistributing excessive $50 \%$ responses in elicited probabilities. Risk Anal. 22(4), 713-723 (2002)

7. Byrne, M., Thompson, P.: Screening and preventable illness. J. Health Econ. 20, 1077-1088 (2001)

8. Carman, K.G., Kooreman, P.: Probability perceptions and preventive health care. J. Risk Uncertain. 49, 43-71 (2014)

9. Dervaux, B., Eeckhoudt, L.: Prévention en économie et en médecine. Revue Economique. 55(5), 849-856 (2004)

10. Egan, M., Philipson, T.: Health Care Adherence and Personalized Medicine. NBER Working Paper no. 20330 (2014)

11. Etner, J., Jeleva, M.: Risk perception, prevention and diagnostic tests. Health Econ. 22, 144-156 (2013)

12. Fang, H., Wang, Y.: Estimating dynamic discrete choice models with hyperbolic discounting, with an application to mammography decisions. NBER: Working Paper 16438 (2010)

13. Fels, M.: The value of medical diagnosis: why people reject medical information. University of Bonn, Mimeo. http://ssrn. com/abstract=1930926 (2012)

14. Finkelstein, A., Luttmer, E.F.P., Notowidigdo, M.J.: Approaches to estimating the health state dependence of the utility function. Am. Econ. Rev. 99, 116-121 (2009)

15. Hadler, J.L., Konty, K., McVeigh, K.H., Fine, A., Eisenhower, D., Kerker, B., Thorpe, L.: Case fatality rates based on population estimates of influenza-like illness due to novel H1N1 influenza: New-York City. PloS One. 5(7), 1-8 (2010)

16. Howard, D.: Life expectancy and the value of early detection. J. Health Econ. 24, 891-906 (2005)

17. Juckett, D.A., Rosenberg, B.: Comparison of the Gompertz and Weibull functions as descriptors for human mortality distributions and their intersections. Mech. Ageing Dev. 69(1), 1-31 (1993)

18. Jusot, F., Or, Z., Sirven, N.: Variations in preventive care utilisation in Europe. Eur. J. Ageing 9, 15-25 (2012)

19. Lamiraud, K., Geoffard, P.: Therapeutic non adherence: a rational behaviour revealing patient preferences. Health Econ. 16(11), 1185-1204 (2007)

20. Listl, S.: Income-related inequalities in dental service utilization by Europeans aged 50+. J. Dent. Res. 90(6), 717-723 (2011)

21. Listl, S., Moran, V., Maurer, J., Faggion Jr, C.M.: Dental service utilization by Europeans aged 50 plus. Commun. Dent. Oral Epidemiol. 40, 164-174 (2012)

22. Maurer, J.: Who has a clue to preventing the flu? Unravelling supply and demand effects on the take-up of influenza vaccinations. J. Health Econ. 28, 704-717 (2009)
23. Mullahy, J.: It'll only hurt a second? Microeconomic determinants of who gets flu shots. Health Econ. 8, 9-24 (1999)

24. National Center for Health Statistics: Health, United States, 2013: With Special Feature on Prescription Drugs. DHHS Publication no. 2014-1232 (2014)

25. Oster, E., Shoulson, I., Dorsey, R.: Optimal expectations and limited medical testing: evidence from Huntington disease. NBER: Working Paper 17629 (2011)

26. Peracchi, F., Perotti, V.: Subjective survival probabilities and life tables: evidence from Europe. Mimeo. http://www.eief.it/files/ 2012/09/peracchi-perotti-2012_dr.pdf (2012)

27. Phelps, C.: Good technologies gone bad: why and how the cost effectiveness of various medical interventions changes for different populations. Med. Decis. Mak. 17(1), 107-112 (1997)

28. Picone, G., Sloan, F., Taylor, D.: Effects of risk and time preference and expected longevity on demand for medical tests. J. Risk Uncertain. 28(1), 39-53 (2004)

29. Schmitz, H., Wübker, A.: What determines influenza vaccination take-up of elderly Europeans? Health Econ. 20, 1281-1297 (2011)

30. Smith, V., Taylor, D., Sloan, F.: Longevity expectations and death: can people predict their own demise? Am. Econ. Rev. 91(4), 1126-1134 (2001)

31. Thompson, M.G., Shay, D.K., Zhou, H., Bridges, C.B., Cheng, P.Y., Burns, E., Bresee, J.S., Cox, N.J.: Updated estimates of mortality associated with seasonal influenza-United States, 1976-2007. Morb. Mortal. Wkly. Rep. 59(33), 1057-1062 (2010)

32. VENICE: http://venice.cineca.org/ (2014). Accessed 1 Oct 2014

33. Viscusi, W.K., Hakes, J.: Risk ratings that do not measure probabilities. J. Risk Res. 6(1), 23-43 (2003)

34. von Karsa, L., Anttila, A., Ronco, G., Ponti, A., Malila, N., Arbyn, M., Segnan, N., Castillo-Beltran, M., Boniol, M., Ferlay, J., Hery, C., Sauvaget, C., Voti, L., Autier, P.: Cancer Screening in the European Union. Report on the implementation of the Council Recommendation on cancer screening, p. 136. European Communities (2008)

35. Weon, B.M.: Analysis of trends in human longevity by new model. Mimeo. http://arxiv.org/ftp/q-bio/papers/0402/0402011. pdf (2004)

36. WHO Globocan: http://globocan.iarc.fr/Pages/DataSource_and_ methods.aspx (2014). Accessed 1 Oct 2014

37. Whynes, D., Philips, Z., Avis, M.: Why do women participate in the English cervical cancer screening programme? J. Health Econ. 26, 306-325 (2007)

38. Widström, E., Eaton, K.A.: Oral healthcare systems in the extended European union. Oral Health Prev. Dent. 2(3), 155-194 (2004)

39. Wilson, D.L.: The analysis of survival (mortality) data: fitting Gompertz, Weibull, and logistic functions. Mech. Ageing Dev. 74(1), 15-33 (1994)

40. Witt, J.: The effect of information in the utilization of preventive health-care strategies: an application to breast cancer. Health Econ. 17, 721-731 (2008)

41. Wu, S.: Sickness and preventive medical behavior. J. Health Econ. 22, 675-689 (2003)

42. Wübker, A.: Explaining variations in breast cancer screening across European countries. Eu. J. Health Econ. 15(5), 497-514 (2014)

43. Wübker, A.: Who gets a mammogram amongst European women aged 50-69 years? Health Econ. Rev. 2(6), 1-13 (2012) 